\title{
A NOTE ON THE LEAST (NORMALIZED) LAPLACIAN EIGENVALUE OF SIGNED GRAPHS
}

\author{
HUI-SHU LI AND HONG-HAI LI*
}

\begin{abstract}
Let $\Gamma=(G, \sigma)$ be a connected signed graph, and $L(\Gamma)$ be its Laplacian and $\mathscr{L}(\Gamma)$ its normalized Laplacian with eigenvalues $\lambda_{1} \geq \lambda_{2} \geq \cdots \geq \lambda_{n}$ and $\mu_{1} \geq \mu_{2} \geq \cdots \geq \mu_{n}$, respectively. It is known that a signed graph $\Gamma$ is balanced if and only if $\lambda_{n}=0$ (or $\mu_{n}=0$ ). We show that $\lambda_{n}$ and $\mu_{n}$ measure how much $\Gamma$ is far from being balanced by proving that

$$
\begin{aligned}
& \mu_{n}(\Gamma) \leq \min \left\{\frac{2 \epsilon(\Gamma)}{m}, \frac{v(\Gamma)}{v(\Gamma)+v_{1}(\Gamma)}\right\}, \\
& \lambda_{n}(\Gamma) \leq \min \left\{\lambda_{1}\left(\Gamma^{\prime}\right): \Gamma-\Gamma^{\prime} \text { is balanced }\right\}
\end{aligned}
$$

where $v(\Gamma)$ (resp. $\epsilon(\Gamma)$ ) denotes the frustration number (resp. the frustration index) of $\Gamma$, that is the minimum number of vertices (resp. edges) to be deleted such that the signed graph is balanced.
\end{abstract}

\section{Introduction}

A signed graph $\Gamma=(G, \sigma)$ consists of an underlying graph $G=(V, E)$ and a mapping $\sigma$ : $E \rightarrow\{+1,-1\}$, where $V:=\left\{v_{1}, v_{2}, \cdots, v_{n}\right\}$ and edge $v_{i} v_{j}$ is denoted by $e_{i j}$. Let $d_{G}(v)$ denote the vertex degree of $v$ in $G$ and for simplicity $d_{G}(v)$ is written as $d_{v}$ when the graph $G$ can be understood from the context.

Signed graphs were introduced by Heider [9] in 1946 to describe sentiment relations between people pertaining to a same social group and to provide a systematic statement of social balance theory. Cartwright and Harary [4] formalized Heider's theory stating that a balanced social group, i.e., a balanced signed graph, could be partitioned into two mutually hostile subgroups each having internal solidarity. Due to its applications in social network,

Received June 3, 2015, accepted January 10, 2016.

2010 Mathematics Subject Classification. 05C50.

Key words and phrases. Signed graph, Laplacian, balancedness, algebraic frustration.

Corresponding author: Hong-Hai Li.

*Supported by National Natural Science Foundation of China (11561032, 11201198), Natural Science Foundation of Jiangxi Province (20142BAB211013), the scientific funds of the Education Department of Jiangxi Province (No. GJJ150345), the Sponsored Program for Cultivating Youths of Outstanding Ability in Jiangxi Normal University. 
portfolio analysis in risk management, biological systems and so on, in which the common element among these applications is the fact that all of them are defined in a collaborative vs. conflicting environment that can be modeled over a signed graph, signed graphs have continued to be a very attractive discrete structure studied extensively.

Let $C$ be a cycle of a signed graph $\Gamma=(G, \sigma)$. The sign of $C$ is denoted by $\operatorname{sgn}(C)=$ $\prod_{e \in C} \sigma(e)$. A cycle whose sign is + (or - ) is called positive (or negative). A signed graph is called balanced if all its cycles are positive. Suppose $\theta: V \rightarrow\{+1,-1\}$ is any sign function. Switching $\Gamma$ by $\theta$ means forming a new signed graph $\Gamma^{\theta}=\left(G, \sigma^{\theta}\right)$ whose underlying graph is the same as $G$, but whose sign function is defined by $\sigma^{\theta}\left(e_{i j}\right)=\theta\left(v_{i}\right) \sigma\left(e_{i j}\right) \theta\left(v_{j}\right)$. Let $\Gamma_{1}=\left(G, \sigma_{1}\right)$ and $\Gamma_{2}=\left(G, \sigma_{2}\right)$ be two signed graph with the same underlying graph $G$. $\Gamma_{1}$ and $\Gamma_{2}$ are called switching equivalent, written $\Gamma_{1} \simeq \Gamma_{2}$, if there exists a switching function $\theta$ such that $\Gamma_{2}=\Gamma_{1}^{\theta}$. Switching leaves many signed-graphic characteristics invariant, such as the set of positive cycles.

The Laplacian matrix $L:=L(\Gamma)=\left(L_{u v}\right)$ of a signed graph $\Gamma$ of order $n$ is the $n \times n$ matrix $L$ defined as follows:

$$
L_{u v}= \begin{cases}d_{u} & \text { if } u=v \\ -\sigma(u v) & \text { if } u v \in E(\Gamma) \\ 0 & \text { otherwise. }\end{cases}
$$

Hou et al.[11] described $L(\Gamma)$ by means of its quadratic form:

$$
x^{T} L(\Gamma) x=\sum_{v_{i} v_{j} \in E(\Gamma)}\left(x_{i}-\sigma\left(v_{i} v_{j}\right) x_{j}\right)^{2},
$$

where $x=\left(x_{1}, \ldots, x_{n}\right)^{T} \in \mathbb{R}^{n}$.

Lemma 1.1 ([17]). Let $\Gamma$ be a connected signed graph and $L(\Gamma)$ be its Laplacian matrix. Then $\Gamma$ is balanced if and only if $\operatorname{det}(L(\Gamma))=0$.

Two matrices $M_{1}$ and $M_{2}$ of order $n$ are called signature similar if there exists a signature matrix, that is, a diagonal matrix $S=\operatorname{diag}\left(s_{1}, s_{2}, \ldots, s_{n}\right)$ with diagonal entries $s_{i}= \pm 1$ such that $M_{2}=S M_{1} S$.

Lemma $1.2([11])$. Let $\Gamma_{1}=\left(G, \sigma_{1}\right)$ and $\Gamma_{2}=\left(G, \sigma_{2}\right)$ be signed graphs on the same underlying graph $G$. Then $\Gamma_{1} \simeq \Gamma_{2}$ if and only if $L\left(\Gamma_{1}\right)$ and $L\left(\Gamma_{2}\right)$ are signature similar.

The normalized Laplacian of $\Gamma$ is the $n \times n$ matrix $\mathscr{L}:=\mathscr{L}(\Gamma)=\left(\mathscr{L}_{u v}\right)$ given by

$$
\mathscr{L}_{u v}= \begin{cases}1 & \text { if } u=v \text { and } d_{u} \neq 0, \\ -\sigma(u v) \frac{1}{\sqrt{d_{u} d_{v}}} & \text { if } u v \in E(\Gamma), \\ 0 & \text { otherwise. }\end{cases}
$$


Let $D$ denote the diagonal matrix with the $(u, u)$ th entry having value $d_{u}$. For a general graph, we have $\mathscr{L}=D^{-1 / 2} L D^{-1 / 2}$ with the convention that $D^{-1}(u, u)=0$ if $d_{u}=0$, where $A=\left(\sigma(u v) a_{u v}\right)$ is the adjacency matrix of $\Gamma$ (i.e., $a_{u v}=1$ if $u$ is adjacent to $v$, and 0 otherwise). By the quadratic form of Laplacian $L(\Gamma)$ and normalized Laplacian $\mathscr{L}(\Gamma)$, they are positive semi-definite and so their eigenvalues are nonnegative and denoted by $\lambda_{1}(\Gamma) \geq \lambda_{2}(\Gamma) \geq \cdots \geq$ $\lambda_{n}(\Gamma) \geq 0$ and $\mu_{1}(\Gamma) \geq \mu_{2}(\Gamma) \geq \cdots \geq \mu_{n}(\Gamma) \geq 0$, respectively.

The following characterization of balanced signed graphs is well known [17, 12, 14].

Lemma 1.3. Let $\Gamma=(G, \sigma)$ be a signed graph. Then the following conditions are equivalent:

(1) $\Gamma$ is balanced.

(2) $\Gamma=(G, \sigma) \sim(G,+)$, where $(G,+)$ is the signed graph with all positive edges.

(3) $V(\Gamma)$ can be partitioned into two color classes $V_{1} \cup V_{2}$ such that every edge between $V_{1}$ and $V_{2}$ is negative and every edge within $V_{1}$ or $V_{2}$ is positive.

(4) $\lambda_{n}(\Gamma)=0$.

(5) $\mu_{n}(\Gamma)=0$.

The Maximum Balanced Subgraph Problem (MBSP) is the problem of finding a subgraph of a signed graph that is balanced and maximizes the cardinality of its vertex set. The MBSP is known to be an NP-hard problem although the problem of detecting balance in signed graphs can be solved in polynomial time. Figueiredo and Frota [6] first discussed applications of the MBSP and the efficient solution of the MBSP. In [2], some parameters called frustration number and frustration index measuring how far a signed graph is to be balanced have been investigated in terms of the least eigenvalue of Laplacian of signed graph. More results on the spectra of signed graphs can be found in $[1,2,3,6,11,12,16,13,14,5,7,8]$.

In this paper, we consider the corresponding problem to bound frustration number and frustration index of signed graphs in terms of its least eigenvalue of normalized Laplacian of signed graphs. Further, we show that the least eigenvalue of Laplacian can be bounded up using the spectral radius of edge-induced subgraph whose deletion makes it being balanced.

\section{Main results}

As in [2], the frustration number $v(\Gamma)$ and the frustration index $\epsilon(\Gamma)$ of signed graph $\Gamma$ are defined as the smallest number of vertices and edges whose deletion leads to a balanced graph, respectively. It is known that $v(\Gamma) \leq \epsilon(\Gamma)$ always holds. In [2], it is shown that $\lambda_{n}(\Gamma) \leq$ $\epsilon(\Gamma)$ (proved first in [16]) and $\lambda_{n}(\Gamma) \leq v(\Gamma)$. We would mention here that $\lambda_{n}(\Gamma)$ has been called the "algebraic frustration". It is in analogy with the algebraic connectivity and algebraic bipartiteness. 
Some notations are needed here. For a signed graph $\Gamma$ with two proper vertex subset $S, T \subseteq V(\Gamma)$, let $E_{\Gamma}(S, T)\left(E_{\Gamma}^{ \pm}(S, T)\right)$ be the set of (positive or negative) edges with one end in $S$ and another end in $T$, and the subscript $\Gamma$ will usually be dropped off when it can be understood from the context. When $S=T, E_{\Gamma}(S, T)\left(E_{\Gamma}^{ \pm}(S, T)\right)$ will be denoted by $E_{\Gamma}(S)\left(E_{\Gamma}^{ \pm}(S)\right)$. Denote by $G[S]$ the induced subgraph of $G$ by $S$. Let $\operatorname{Vol}(G)$ denote the sum of the degrees of all vertices in $G$.

If $\Gamma$ is unbalanced, then there exists a signed subgraph $S \subset \Gamma$, with $|S|=v(\Gamma)$, such that $\Gamma-S$ is balanced. Note that $\Gamma-S$ might be not connected, say $\Gamma-S=S_{1}+\cdots+S_{k}$ is balanced where each $S_{i}$ is a connected balanced signed subgraph of $\Gamma$. It is easy to see that $|S| \leq n-2$. Further, in this case, define $\psi(S)=\max _{1 \leq i \leq k} \frac{\operatorname{Vol}\left(G\left[S_{i}\right]\right)}{\left|S_{i}\right|}$. Maximizing $\psi(S)$ over all such $S$ such that $|S|=v(\Gamma)$ and $\Gamma-S$ is balanced, we get a parameter and denote it by $v_{1}(\Gamma)$.

In what follows, we give our main result that measures how much $\Gamma$ is far from being balanced via frustration number and the frustration index in terms of the least eigenvalue of normalized Laplacian.

Theorem 2.1. Let $\Gamma=(G, \sigma)$ be a signed graph with $n$ vertices and $m$ edges. Then $\mu_{n}(\Gamma) \leq$ $\min \left\{\frac{2 \epsilon(\Gamma)}{m}, \frac{v(\Gamma)}{v(\Gamma)+v_{1}(\Gamma)}\right\}$

Proof. If $\Gamma$ is balanced, then $\mu_{n}(\Gamma)=0$ and $v(\Gamma)=\epsilon(\Gamma)=0$, and there is nothing to prove. Then suppose that $\Gamma$ is unbalanced thereafter. Let $F \subseteq E(G)$, with $|F|=\epsilon(\Gamma) \geq 0$, be a minimum set of edges for which $\Gamma-F$ is balanced, and let $V_{1}, V_{2}$ be the two color classes of $\Gamma-F$ guaranteed by (3) of Lemma 1.3. Let us define the following vector $X$ on the vertices of $G, X=\left(x_{1}, x_{2}, \cdots, x_{n}\right)^{\top}$ where $x_{i}=1$ if $v_{i} \in V_{1}, x_{i}=-1$ if $v_{i} \in V_{2}$. Observe that $\left(x_{i}-\sigma\left(e_{i j}\right) x_{j}\right)^{2}=0$ whenever $e_{i j} \in$ $E(G) \backslash F$. In addition, $F=E^{-}\left(V_{1}\right) \cup E^{-}\left(V_{2}\right) \cup E^{+}\left(V_{1}, V_{2}\right)$, so $\left(x_{i}-\sigma\left(e_{i j}\right) x_{j}\right)^{2}=4$ whenever $e_{i j} \in F$.

Hence, let $f=D^{\frac{1}{2}} X$, we have

$$
\begin{aligned}
\mu_{n}(\Gamma) & \leq \frac{f^{T} \mathscr{L}(\Gamma) f}{f^{T} f}=\frac{X^{T} L(\Gamma) X}{X^{T} D X}=\frac{\sum_{e_{i j} \in E(G)}\left(x_{i}-\sigma\left(e_{i j}\right) x_{j}\right)^{2}}{\sum_{v_{i} \in V(G)} d_{i} x_{i}^{2}} \\
& =\frac{\sum_{e_{i j} \in E(G) \backslash F}\left(x_{i}-\sigma\left(e_{i j}\right) x_{j}\right)^{2}+\sum_{e_{i j} \in F}\left(x_{i}-\sigma\left(e_{i j}\right) x_{j}\right)^{2}}{\sum_{v_{i} \in V(G)} d_{i} x_{i}^{2}} \\
& =\frac{2 \epsilon(\Gamma)}{m} .
\end{aligned}
$$

Now, let $S \subseteq V(G)$ with $|S|=v(\Gamma) \leq n-2$ such that $\Gamma-S=S_{1}+\cdots+S_{k}$ is balanced with each $S_{i}$ being connected and balanced. For each balanced component $S_{i}$, again by Lemma 1.3, let $S_{i}^{1}$ be the first color class and $S_{i}^{2}$ the second color class. Define the vector $X_{i}=\left(x_{1}, x_{2}, \ldots, x_{n}\right)$ on $V(\Gamma)$ such that $x_{v}=1$ if $v \in S_{i}^{1}, x_{v}=-1$ if $v \in S_{i}^{2}$, and $x_{v}=0$ if $v \in \Gamma-S_{i}$, for some fixed $1 \leq i \leq k$. Let $\bar{\Gamma}=(\bar{G}, \bar{\sigma})$ be the signed graph obtained from $\Gamma$ such that the underlying graph $\bar{G}$ is obtained from $G$ by adding some edges, say $a$ edges, between $S$ and $S_{i}$, such that each 
vertex in $S$ is adjacent to each vertex in $S_{i}$, and $\bar{\sigma}$ is obtained from $\sigma$ by randomly giving any sign on the added edges.

Note that $E(G) \subseteq E(\bar{G})$, and $\left(x_{u}-\bar{\sigma}(u v) x_{v}\right)^{2}=1$ for any edge of $E_{\bar{G}}\left(S, S_{i}\right)$ and $\left(x_{u}-\bar{\sigma}(u v) x_{v}\right)^{2}=$ 0 otherwise. Similarly, $\left(x_{u}-\sigma(u v) x_{v}\right)^{2}=1$ for any edge of $E_{G}\left(S, S_{i}\right)$ and $\left(x_{u}-\sigma(u v) x_{v}\right)^{2}=0$ otherwise. Since adding each edge between $S$ and $S_{i}$ contributes one to the degree of vertex in $S_{i}$, we have

$$
\begin{aligned}
\mu_{n}(\Gamma) & \leq \frac{X^{T} L(\Gamma) X}{X^{T} D X}=\frac{\sum_{e_{i j} \in E(G)}\left(x_{i}-\sigma\left(e_{i j}\right) x_{j}\right)^{2}}{\sum_{v_{i} \in V(G)} d_{i} x_{i}^{2}}=\frac{\left|E_{G}\left(S, S_{i}\right)\right|}{\sum_{v_{i} \in S_{i}} d_{G}\left(v_{i}\right)} \\
& \leq \frac{\left|E_{G}\left(S, S_{i}\right)\right|+a}{\sum_{v_{i} \in S_{i}} d_{G}\left(v_{i}\right)+a}=\frac{|S| \cdot\left|S_{i}\right|}{\sum_{v_{i} \in S_{i}} d_{\bar{G}}\left(v_{i}\right)}=\frac{|S| \cdot\left|S_{i}\right|}{|S| \cdot\left|S_{i}\right|+\operatorname{Vol}\left(G\left[S_{i}\right]\right)} \\
& =\frac{|S|}{|S|+\frac{\operatorname{Vol}\left(G\left[S_{i}\right]\right)}{\left|S_{i}\right|}}
\end{aligned}
$$

where the second inequality holds because $\left|E_{G}\left(S, S_{i}\right)\right| \leq \sum_{v_{i} \in S_{i}} d_{G}\left(v_{i}\right)$ and the inequality $\frac{c}{d} \leq$ $\frac{c+a}{d+a}$ holds when $c \leq d$. Then the inequality $\mu_{n}(\Gamma) \leq \frac{v(\Gamma)}{v(\Gamma)+v_{1}(\Gamma)}$ follows.

Next we consider the Laplacian matrix of a signed graph $\Gamma=(G, \sigma)$ with $n$ vertices and $m$ edges. For each edge of $e=u v$ of $G$, we choose one of $u$ or $v$ to be the head of $e$ and them other to be the tail. We call this an orientation of $\Gamma$. The incidence matrix $C=C(\Gamma)$ afforded by a fixed orientation of $\Gamma$ is the $n \times m$ matrix $C=\left(c_{\nu e}\right)$, where

$$
c_{v e}= \begin{cases}1 \quad \text { if } v \text { is the head of } e \\ -1 \text { if } v \text { is the tail of } e \text { and } \sigma(e)=+ \\ 1 \quad \text { if } v \text { is the tail of } e \text { and } \sigma(e)=- \\ 0 \quad \text { otherwise. }\end{cases}
$$

Note that the rows and columns of $C$ are indexed by vertices and edges of $\Gamma$, respectively. While $C$ depends on the orientation of $\Gamma, C C^{T}$ does not, and it is easy to verify that $C C^{T}=$ $L(\Gamma)=D(\Gamma)-A(\Gamma)$.

If $\Gamma$ is unbalanced, there exists $F \subseteq E(G)$ such that $|F|=\epsilon(\Gamma)=k$ and $\Gamma-F$ is balanced. Without loss of generality, assume that last columns are indexed by $F$ and so $C$ can be written in the form of $C=\left(C_{1} C_{2}\right)$, where $C_{2}$ is a $n \times \epsilon(\Gamma)$ matrix. Thus we have $L(\Gamma)=C C^{T}=C_{1} C_{1}^{T}+$ $C_{2} C_{2}^{T}$, and let $L_{i}=C_{i} C_{i}^{T}$ for $i=1,2$. Note that $L_{i}$ can be viewed as the Laplacian of signed graph $\Gamma_{i}$ for $i=1,2$, and $\Gamma_{1}$ and $\Gamma_{2}$ be signed subgraphs induced by the edge sets $E(\Gamma)-F$ and $F$, respectively.

Theorem 2.2 ([10]). Let $A, B$ be $n \times n$ Hermitian matrices, and let the eigenvalues of $A, B$ and $A+B$ be arranged in decreasing order $\lambda_{1}(\cdot) \geq \lambda_{2}(\cdot) \geq \cdots \geq \lambda_{n}(\cdot)$. Then for every pair of integers $j, k$ such that $1 \leq j, k \leq n$ and $j+k>1$ we have

$$
\lambda_{j+k-1}(A+B) \leq \lambda_{j}(A)+\lambda_{k}(B) .
$$


Consider the Laplacian $L(\Gamma)=L_{1}+L_{2}$, and we have $\lambda_{n}(\Gamma)=\lambda_{n}(L(\Gamma)) \leq \lambda_{n}\left(L_{1}\right)+\lambda_{1}\left(L_{2}\right)$. By the choice of $F, \Gamma-F$ is balanced and so $\lambda_{n}\left(L_{1}\right)=0$ by Lemma 1.3. Below, we let $\Gamma^{\prime}$ denote signed subgraph induced by some edge set $F$ in $\Gamma$. Thus we come to the following result.

Theorem 2.3. Let $\Gamma$ be a signed graph with n vertices. Then $\lambda_{n}(\Gamma) \leq \min \left\{\lambda_{1}\left(\Gamma^{\prime}\right): \Gamma-\Gamma^{\prime}\right.$ is balanced $\}$.

Note that when $\Gamma$ is balanced, $\Gamma^{\prime}$ is then an empty graph and so $\lambda_{1}\left(\Gamma^{\prime}\right)=0=\lambda_{n}(\Gamma)$.

Now we intend to obtain some upper bound for the spectral radius of Laplacian of signed graphs, which together with the above Theorem 2.3 will imply some upper bound for the least eigenvalue of Laplacian of signed graphs.

For a vertex $v$ of $\Gamma$, denote by $m_{v}=\left(1 / d_{v}\right) \sum_{u v \in E(\Gamma)} d_{u}$, which is called the average 2degree of the vertex $v$. Zhang [19] obtained that $\lambda_{1}(G) \leq \max \left\{d_{i}+\sqrt{d_{i} m_{i}}: v_{i} \in V(G)\right\}$ for unsigned graphs. Next we shall generalize it to signed graphs.

Theorem 2.4. Let $\Gamma=(G, \sigma)$ be a signed graph with $n$ vertices. Then

$$
\lambda_{1}(\Gamma) \leq \max \left\{d_{i}+\sqrt{d_{i} m_{i}} \mid v_{i} \in V(\Gamma)\right\} .
$$

Proof. Let $X=\left(x_{j}, v_{j} \in V(\Gamma)\right)^{T}$ be an eigenvector corresponding to $\lambda_{1}(\Gamma)$. Thus $L(\Gamma) X=$ $\lambda_{1}(\Gamma) X$. Hence for any $v_{i} \in V(\Gamma)$,

$$
\lambda_{1}(\Gamma) x_{i}=d_{i} x_{i}-\sum_{v_{i} v_{j} \in E(\Gamma)} \sigma\left(v_{i} v_{j}\right) x_{j}=\sum_{v_{i} v_{j} \in E(\Gamma)}\left(x_{i}-\sigma\left(v_{i} v_{j}\right) x_{j}\right)
$$

By Cauchy-Schwarz inequality, we have

$$
\begin{aligned}
\lambda_{1}^{2}(\Gamma) x_{i}^{2} & \leq\left(\sum_{v_{i} \nu_{j} \in E(\Gamma)} 1^{2}\right)\left(\sum_{v_{i} v_{j} \in E(\Gamma)}\left(x_{i}-\sigma\left(e_{i j}\right) x_{j}\right)^{2}\right) \\
& =d_{i}^{2} x_{i}^{2}-2 d_{i} x_{i} \sum_{v_{j}: v_{j} \sim v_{i}} \sigma\left(e_{i j}\right) x_{j}+d_{i} \sum_{v_{i} v_{j} \in E(\Gamma)} x_{j}^{2} \\
& =d_{i}^{2} x_{i}^{2}+2 d_{i} x_{i}^{2}\left(\lambda_{1}(\Gamma)-d_{i}\right)+d_{i} \sum_{v_{i} v_{j} \in E(\Gamma)} x_{j}^{2} .
\end{aligned}
$$

Hence

$$
\begin{aligned}
\sum_{v_{i} \in V(\Gamma)} \lambda_{1}^{2}(\Gamma) x_{i}^{2} & \leq \sum_{\nu_{i} \in V(\Gamma)}\left(2 d_{i} \lambda_{1}(\Gamma)-d_{i}^{2}\right) x_{i}^{2}+\sum_{v_{i} \in V(\Gamma)} d_{i} \sum_{v_{i} \nu_{j} \in E(\Gamma)} x_{j}^{2} \\
& =\sum_{v_{i} \in V(\Gamma)}\left(2 d_{i} \lambda_{1}(\Gamma)-d_{i}^{2}\right) x_{i}^{2}+\sum_{v_{i} \in V(\Gamma)} d_{i} m_{i} x_{i}^{2} .
\end{aligned}
$$

Therefore, we have

$$
\sum_{v_{i} \in V(\Gamma)}\left(\lambda_{1}^{2}(\Gamma)-2 d_{i} \lambda_{1}(\Gamma)+d_{i}^{2}-d_{i} m_{i}\right) x_{i}^{2} \leq 0 .
$$


Then there must exist a vertex $v_{i}$ such that

$$
\lambda_{1}^{2}(\Gamma)-2 d_{i} \lambda_{1}(\Gamma)+d_{i}^{2}-d_{i} m_{i} \leq 0,
$$

which implies $\lambda_{1}(\Gamma) \leq d_{i}+\sqrt{d_{i} m_{i}}$ and we are done.

By Theorems 2.3 and 2.4, in most cases the obtained value is less than the frustration index $\epsilon(\Gamma)$, i.e. the edge number of $\Gamma^{\prime}$. For example, if $\Gamma^{\prime}$ is triangle-free and $d_{v} \leq\left|E\left(\Gamma^{\prime}\right)\right|-$ $\sqrt{\left|E\left(\Gamma^{\prime}\right)\right|}$ for every $v \in V\left(\Gamma^{\prime}\right)$, then by Theorem 2.4 we have $\lambda_{1}\left(\Gamma^{\prime}\right) \leq d_{i}+\sqrt{d_{i} m_{i}} \leq\left|E\left(\Gamma^{\prime}\right)\right|-$ $\sqrt{\left|E\left(\Gamma^{\prime}\right)\right|}+\sqrt{\left|E\left(\Gamma^{\prime}\right)\right|}=\left|E\left(\Gamma^{\prime}\right)\right|=\epsilon(\Gamma)$. That is what we get in the following corollary.

Corollary 2.5. Let $\Gamma$ be a signed graph with $n$ vertices and $\Gamma^{\prime}$ is triangle-free and $d_{v} \leq\left|E\left(\Gamma^{\prime}\right)\right|-$ $\sqrt{\left|E\left(\Gamma^{\prime}\right)\right|}$ for every $v \in V\left(\Gamma^{\prime}\right)$. Then $\lambda_{n}(\Gamma) \leq \lambda_{1}\left(\Gamma^{\prime}\right) \leq \epsilon(\Gamma)$.

In this sense, we have obtained a better bound for the least eigenvalue of Laplacian of signed graphs than that $\lambda_{n}(\Gamma) \leq \epsilon(\Gamma)$ in [2].

\section{References}

[1] F. M. Atay and H. Tuncel, On the spectrum of normalized Laplacian for signed graphs: Interlacing, contraction, and replication, Lin. Algebra Appl., 442 (2014), 165-177.

[2] F. Belardo, Balancedness and the least eigenvalue of Laplacian of signed graphs, Lin. Algebra Appl., 446 (2014), 133-147.

[3] F. Belardoa and S. K. Simić, On the Laplacian coefficients of signed graphs, Lin. Algebra Appl., 475 (2015), 94-113.

[4] D. Cartwright and F. Harary, Structural balance: A generalization of heider's theory, Psychological Review, 63 (1956), 277-293.

[5] S. Fallat and Y. Fan, Bipartiteness and the least eigenvalue of signless Laplacian of graphs, Lin. Algebra Appl., 436 (2012), 3254-3267.

[6] R. Figueiredo and Y. Frota, The maximum balanced subgraph of a signed graph: Applications and solution approaches, European Journal of Operational Research, 236 (2014), 473-487.

[7] K. A. Germina, S. Hameed and K. T. Zaslavsky, On products and line graphs of signed graphs, their eigenvalues and energy, Lin. Algebra Appl., 435 (2011), 2432-2450.

[8] G. Greaves, J. Koolen, A. Munemasa, Y. Sano and T. Taniguchi, Edge-signed graphs with smallest eigenvalue greater than -2, Journal of Combinatorial Theory, Series B, 110 (2015), 90-111.

[9] F. Heider, Attitudes and cognitive organization, Journal of Psychology, 21 (1946), 107-112.

[10] R. A. Horn and C. R. Johnson, Matrix Analysis, Cambridge University Press, Cambridge, UK, 1985.

[11] Y. Hou, J. Li and Y. Pan, On the Laplacian eigenvalues of signed graphs, Linear and Multilinear Alg., 51 (2003), 21-30.

[12] Y. Hou, Bounds for the least Laplacian eigenvalues of a signed graphs, Acta Mathematica Sinica (Engl. Ser.), 21 (2005), 955-960.

[13] H. Li and J. Li, An upper bound on the Laplacian spectral radius of the signed graphs, Discuss. Math. Graph Theory, 28 (2008), 345-359.

[14] H. Li and J. Li, Note on the normalized Laplacian eigenvalues of signed, Australasian Journal of Combinatorics, 44 (2009), 153-162.

[15] Y. Liu and J. Shen, The (normalized) Laplacian eigenvalue of signed graphs, Taiwanese Journal of Mathematics, in press. 
[16] Y. Tan and Y. Fan, On edge singularity and eigenvectors of mixed graphs, Acta Mathematica Sinica (Engl. Ser.), 24 (2008), 139-146.

[17] T. Zaslavsky, Signed graphs, Discrete Appl. Math., 4 (1982), 47-74.

[18] X. Zhang and R. Luo, The Laplacian eigenvalues of mixed graphs, Lin. Algebra Appl., 362 (2003), $109-119$.

[19] X. Zhang, Two sharp upper bounds for the Laplacian eigenvalues, Linear Algebra Appl., 376 (2004), $207-213$.

College of Mathematics and Information Science, Jiangxi Normal University, Nanchang, JiangXi, 330022, People's Republic of China.

E-mail: lhh@jxnu.edu.cn 